\title{
Onset and Peak Pattern Recognition on Photoplethysmographic Signals Using Neural Networks
}

\author{
Alvaro D. Orjuela-Cañón ${ }^{1}$, Denis Delisle-Rodríguez ${ }^{2}$, Alberto López-Delis ${ }^{2}$, \\ Ramón Fernandez de la Vara-Prieto ${ }^{2}$, and Manuel B. Cuadra-Sanz ${ }^{3}$ \\ ${ }^{1}$ GIBIO - Electronic and Biomedical Faculty, Universidad Antonio Nariño, \\ Bogotá D.C., Colombia \\ alvorjuela@uan. edu.co \\ 2 Center of Medical Biophysics, Universidad de Oriente, \\ Santiago de Cuba, Cuba \\ \{denis.delisle,ramon.fernandez, alberto.lopez\}@cbiomed.cu \\ 3 CIDEI (Research and Technologic Development Center for the Electro-Electronics \\ and Informatics Industry), \\ Bogotá D.C., Colombia \\ mqadra20130yahoo.es
}

\begin{abstract}
Traditional methodologies use electrocardiographic (ECG) signals to develop automatic methods for onset and peak detection on the arterial pulse wave. In the present work a Multilayer Perceptron (MLP) neural network is used for classifying fiducial points on photoplethysmographic (PPG) signals. System was trained with a dataset of temporal segments from signals located based on information about onset and peak points. Different segments sizes and units in the neural network were used for the classification, and optimal values were searched. Results of the classification reach $98.1 \%$ in worse of cases. This proposal takes advantages from MLP neural networks for pattern classification. Additionally, the use of ECG signal was avoided in the presented methodology, making the system robust, less expensive and portable in front of this problem.
\end{abstract}

Keywords: Arterial Pulse Wave, Artificial Neural Networks, Multilayer Perceptron, Onset Classification, Peak Classification.

\section{Introduction}

The photoplethysmography (PPG) signal has been used as a simple and low-cost optical technique, which is used for measuring blood volume changes through of the light intensity during the emission and reception on the skin surface. Peripheral body sites such as fingers, ears, toes and forehead are used to obtain these kind of signals, approaching blood volume and perfusion changes due to the dissemination or absorption of the incident light, providing the dynamical part of the signal $[12]$.

J. Ruiz-Shulcloper and G. Sanniti di Baja (Eds.): CIARP 2013, Part I, LNCS 8258, pp. 543-550, 2013.

(C) Springer-Verlag Berlin Heidelberg 2013 
Applications of PPG signal treatment can be seen in commercial medical equipment, where measures of oxygen saturation, blood pressure or heart rate monitoring assess autonomic functions and contributes to peripheral vascular diseases diagnosis. In this way, fiducial points detection on PPG signals is used to obtain relevant information such as pulse transit time (PTT) and pulse wave velocity $(\mathrm{PWV})$, which evaluate vascular effects of aging, hypertension, stiffness and atherosclerosis 34 .

Onset and peak pulses detection is not a trivial task, due to natural conditions as sensor positioning, skin features, breathing, baseline drift, perfusion phenomena, visco-elastic and viscosity property of arteries, arterial stiffness and reflected waves from peripheral sites, which can be easily involved in noise and artifacts. As PPG signal typically has small amplitude, its incident and reflected waveform can be affected for these kind of conditions [5].

Different methods have been developed for this detection task varying its complexity. These can include adaptive threshold, computer-based filtering, feature extraction, and derivative calculation 6 67]. Most of them are assisted by the electrocardiographic (ECG) signal, which provides a cost increment of medical equipment and difficult its clinical applications in the Health Primary System. In 7], morphological similarity of adjacent pulse is used to enhance signal quality and increase the accuracy of the onset pulses detection. A disadvantage of the method is the inclusion of measures from time interval between $R$ to $R$ peak of ECG signal. Additionally, it is applied principal components analysis over adjacent peaks to enhance the onset detection. Information about diastolic point, second derivative and tangent intersection, shows an enhanced accuracy and precision [8]. The use of ECG signal problem, again represents a disadvantage when is not possible obtain this kind of signals. Recently, in [9] a new method is presented, based on filed collected photoplethysmograms. This method does not use ECG signal and works through PPG signal filtering in different ways, but digital filters introduce delays in the temporal signal, which can give wrong information about onset localization in signal. Other function of that algorithm is the detection of peak pulses in the PPG signal.

Neural networks has been used in cardiovascular problems detection, such as QRS detection [1011], clustering 1213] and applications with PPG signals 14 15. Despite its advantages for pattern recognition, few studies have been reported as alternative method for onset and peak pulses detection. An example of this can be found in [16], where Self Organizing Maps (SOM) were employed for the onset and peak detection and classification.

In this paper, it is presented a proposal based on pattern recognition, which uses a Multilayer Perceptron (MLP) to learn the temporal information around onset and peak pulses. Supervised training is implemented, where PPG signals marked by expert observers are used. Onset and peak are designated into a temporal windows used to train the neural network. For validation of the models, the Leave One Out cross validation method is implemented, which is used when the database is the limited size. 


\section{Materials and Methods}

Database is composed by signals from seven volunteers, who participated in the experimental protocol and provide informed consent in accordance with institutional policy. Each subject remains in rest during five minutes in the supine position. Previously to the test, personal and clinical data were collected of each volunteer. Table 1 shows the age and vital signs of the subjects.

Table 1. Characteristics of Volunteers

\begin{tabular}{ccccccc}
\hline Subject & Sex & Age(years) & HR $(\mathrm{bpm})$ & SBP $(\mathrm{mmHg})$ & $\mathrm{DBP}(\mathrm{mmHg})$ & Breath $/ \mathrm{min}$ \\
\hline 1 & $\mathrm{M}$ & 19 & 72 & 102 & 68 & 20 \\
2 & $\mathrm{M}$ & 20 & 60 & 98 & 60 & 16 \\
3 & $\mathrm{M}$ & 22 & 80 & 125 & 80 & 17 \\
4 & $\mathrm{M}$ & 20 & 84 & 112 & 72 & 18 \\
5 & $\mathrm{~F}$ & 18 & 80 & 110 & 64 & 16 \\
6 & $\mathrm{~F}$ & 18 & 88 & 108 & 58 & 22 \\
7 & $\mathrm{~F}$ & 18 & 72 & 108 & 68 & 19 \\
Mean \pm SD & & $19.3 \pm 1.50$ & $76.57 \pm 9.36$ & $109 \pm 8.54$ & $67.14 \pm 7.47$ & $18.28 \pm 2.21$ \\
\hline
\end{tabular}

HR: Heart rate

SBP: Systolic blood pressure

DBP: Diastolic blood pressure

SD: Standard deviation

Acquisition was performed by an experimental station, which collected physiological signals of each person. This station simultaneously acquires electrocardiography (ECG) and photoplethysmographic (PPG) signals through an ECG channel with bandwidth between 0.15 to $150 \mathrm{~Hz}$ and two PPG channels with bandwidth from 0.5 to $16 \mathrm{~Hz}$. Simultaneous ECG and PPG signals were sampled with $1000 \mathrm{~Hz}$ and manually marked by trained observers from Center of Medical Biophysics. This task was developed using functions in Matlab software (Mathworks Inc., Natick, MA), which provide additional capabilities such as, add, move and remove the fiducial points of ECG and PPG signals (Fig. 11). These points correspond to peaks values of QRS complex and, onsets and peaks of PPG signal. This information is useful for testing the proposed method.

\subsection{Neural Networks Training}

Neural networks have the ability of learning complex nonlinear patterns, based on input-output relationships, adjusting a set of free parameters known as synaptic weights. The most common type of networks employed in classification tasks are the Multilayer Perceptrons (MLPs), which have only feed forward connections and are trained in a supervised way [17.

In the present work, MLPs are employed to learn patterns in temporal segments around onset and peak fiducial points on PPG signal. This work is based on the annotations of the expert observers, which marked the onset and peak 




Fig. 1. PPG signal acquisition and annotation system

points. These fiducial points are located in the middle of segment, and then is extracted the temporal segment (Figure 2). The segment size is a topic of study in the present study, then windows with values of $21,41,61,81$ and 101 milliseconds were used and observed its relation with the behaviour of the network.

Smaller sizes were not considered because are shorter windows to pattern representation and can be confused with noise segments. In PPG signals, distance between onset and peak is less than 100 milliseconds, due to its nature [1819]. For this reason, an upper period of time was not considered. Windows with the same size of the onset and peak pattern were extracted to create a third class called noise, these segments were taken from samples before and after of the onset and peak windows. This makes that the noise represents segments of signal without activity involved in other classes.

MLP architecture consists of an input, a hidden layer and a output layer. Number of nodes in the input is given by the segment size; number of units in the hidden layer was heuristically studied, trying 5, 10, 15 and 20 units. In the output layer were used three units, each one identifies each pattern. More layers are not used because two layers are enough for a typical problem of classification [17.

Each segment is normalized before being presented to the network, maintaining its values in the interval from 0 to 1 . This contributes to avoid the saturation of the values in the synaptic weights, which are initialized in a random manner. Hyperbolic tangent functions are used in each unit as activation functions. In this way, the units in the output have the value of one when the input belongs to this class, and -1 when does not belong. Training for MLP is developed in 
a supervised manner, where patterns and its labels are necessary. The resilient backpropagation algorithm was used for the training of the network, due to its fast and stable convergence 20. Training set must represent the statistics of the data, and its construction is important for a good training, because the network must generalize its performance to new inputs.

In the present study, the database has a limited size, making difficult the division of the data for training and validation. Alternatives as bootstrapping and cross validation methods can be useful in these cases. An example of this kind of methods is the Leave One Out (LOO) method, where the performance of pattern classification models can be evaluated based on LOO error. The applied method consists in training the network with all data, leaving only an observation out. In the present case, six of seven signals were used in the training, each of them with 345 onset and 345 peak points. When training was finished, the validation is calculated using just with the signal not included in the training, also with 345 onset and peak points. Finally, there is many models as observations of database. For this reason the method is employed with databases with limited observations.

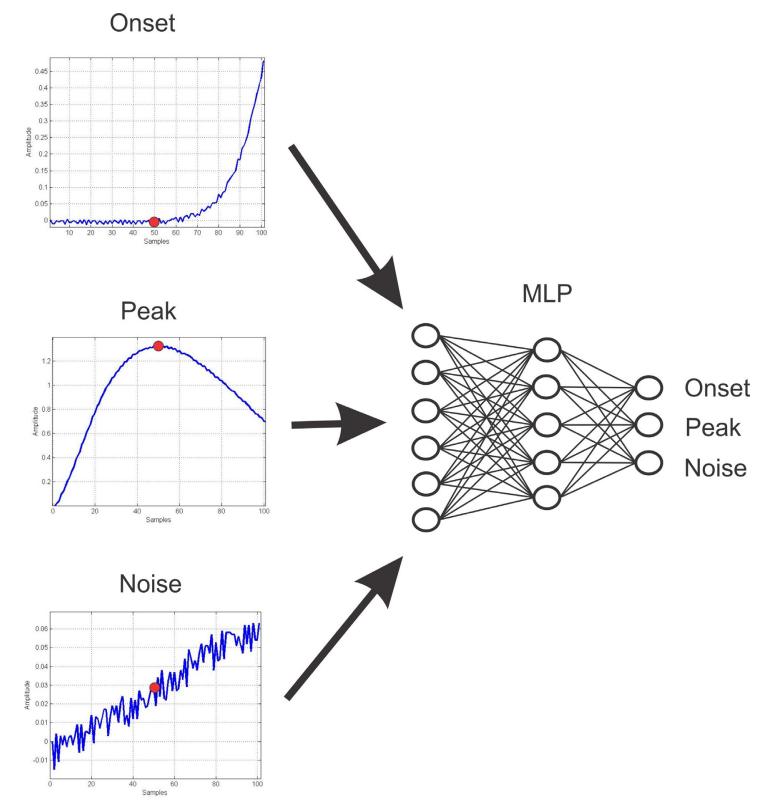

Fig. 2. Onset, peak and noise patterns and MLP Neural Network

The LOO error is a statistical estimator of the behaviour when a learning algorithm is used, and it is very useful for model selection because is slightly biased, despite its empirical error. Also, when the algorithm is stable, LOO error is low [2122]. The LOO error can be calculated by using:

$$
E_{L O O}\left(f_{D}\right)=\frac{1}{m} \sum_{i=1}^{m} \ell\left(f^{i}, z_{i}\right)
$$


where $m$ is the number of samples in the $D$ set, composed by the $z_{i}$ elements, and it is built the learning algorithm, $f^{i}$ is the function obtained after training.

These methods have been used in applications where models of regression or structures in time series are required. Also, there have been in areas such as digital signal processing, obtaining good results 2324 .

\section{Results and Discussion}

As mentioned, windows with 21, 41, 61, 81 and 101 milliseconds were used to represent the patterns of the fiducial points and networks with 5, 10, 15 and 20 units in the hidden layer were trained. The results are presented in the Table 2 , where the error was calculated based on expression (1). The results represent the performance of the MLP method for classification, obtaining seven networks due to the validation methodology, where for each window size and number of units was trained one network. Average performance of the technique was computed using the result of each of these networks.

The results show that models with few units in the hidden layer are preferred, because when the number of units grows up the network is more specialized, decreasing the performance. Results for networks with 21, 41 and 61 milliseconds manifest this behavior, where the best results were given when the network just has five units in the hidden layer.

Window size used for the pattern representation is relevant for the obtained models. When sizes of 81 and 101 milliseconds are used, the results reach the maximum possible, showing that neural networks can learn the patterns extracted from PPG signal. It is important to note that large networks are avoid, because the computational cost is higher and its training can be more specialized. In this way, the best model to develop the pattern classification is a network with five units in the hidden layer and 61 nodes in the input.

Table 2. Results for Classification

\begin{tabular}{ccccc}
\hline \hline Window & \multicolumn{4}{c}{ Units in the hidden layer } \\
\cline { 2 - 5 } size $(\mathrm{ms})$ & 5 & 10 & 15 & 20 \\
\hline 21 & $98.10 \pm 1.31$ & $97.79 \pm 1.41$ & $97.78 \pm 1.46$ & $97.94 \pm 1.45$ \\
41 & $99.92 \pm 0.14$ & $99.87 \pm 0.27$ & $99.90 \pm 0.15$ & $99.89 \pm 0.19$ \\
61 & $100 \pm 0$ & $99.96 \pm 0.06$ & $99.96 \pm 0.06$ & $99.96 \pm 0.06$ \\
81 & $100 \pm 0$ & $100 \pm 0$ & $100 \pm 0$ & $100 \pm 0$ \\
101 & $100 \pm 0$ & $100 \pm 0$ & $100 \pm 0$ & $100 \pm 0$ \\
\hline \hline
\end{tabular}

These results compared with the used method in [16], where a Self Organizing Map (SOM) was implemented to do the classification, are quite close in terms of accuracy. There, accuracy values reach $97.93 \%$ using a map of 36 x 10 units in lattice, a Gaussian function as neighborhood function and 2000 iterations. The noise class for the SOM case was not defined, detecting just onset and peak 
segments. Those results can be compared with accuracy of $100 \%$ in this work, but validation for the SOM case was developed in a hold out manner.

In addition, it is important to say that the present study does not use the ECG signal for detection, which is a considerable difference due to the smaller amount of information that contains just the PPG signal. This is taken as advantage when the resources are restricted to develop a detection.

\section{Conclusions}

A proposal for onset and peak pattern classification has been presented, based on MLP networks. Capabilities of this kind of neural networks are exploited to learn onset and peak patterns on PPG signals, using a temporal window as input.

Parameters as the number of units in the hidden layer and the segment size used in the network input were studied. Results show that the best performance can be reach using 61 milliseconds in the temporal window and a neural network with five units in the hidden layer, when the accuracy is $100 \%$.

The results show that the MLP neural network can be used to develop a detector using a sliding temporal window on PPG signals, taking advantage of capabilities from MLP as pattern classifier. The presented work can be complemented in this way, avoiding the use of ECG signal for the detection. Some additional studies can improve the method and obtain better results.

Acknowledgments. This work was supported under grant: PI/UAN-2013566GB from Universidad Antonio Nariño, Colombia. Also, authors want to thank to Universidad de Oriente for the support in this work.

\section{References}

1. Allen, J.: Photoplethysmography and its application in clinical physiological measurement. Physiol. Meas. 28, 1-39 (2007)

2. Challoner, A.V.J.: Photoelectric plethysmography for estimating cutaneous blood flow. In: Rolfe, P. (ed.) Non-Invasive Physiological Measurements, vol. 1, pp. 125130. Academic, London (1979)

3. Willum-Hansen, T., Staessen, J.A., Torp-Pedersen, C., Rasmussen, S., Thijs, L., Ibsen, H., Jeppesen, J.: Prognostic value of aortic pulse wave velocity as index of arterial stiffness in the general population. Circulation 113, 664-670 (2006)

4. Bistra, N., Ivo, I.: An automated algorithm for fast pulse wave detection. Bioautomation 14(3), 203-216 (2010)

5. Lyengar, N., Peng, C.K., Morin, R., Goldberger, A.L., Lipsitz, L.A.: Age-related alterations in the fractal scaling of cardiac interbeat interval dynamics. American Journal of Physiology (Regulation, Integration and Comparative Physiology) 271, R1078-R1084 (1996)

6. Egidijus, K.R.G., Arunas, V.: Mathematical methods for determining the foot point of the arterial pulse wave and evaluation of proposed methods. Inform. Technol. and Control 3, 29-36 (2005) 
7. Xu, P., Bergsneider, M., Hu, X.: Pulse onset detection using neighbor pulse-based signal enhancement. Medical Engineering and Physics 31, 337-345 (2009)

8. Hang, S., Chungkeun, L., Myoungho, L.: Adaptive threshold method for the peak detection of photoplethysmographic waveform. Computer in Biology and Medicine 39, 1145-1152 (2009)

9. Liangyou, C., Andrew, T., Jaques, R.: Automated beat onset and peak detection algorithm for field-collected photoplethysmograms. In: IEEE EMBS, pp. 5689-5692 (2009)

10. Markowska-Kacsmar, U., Kordas, B.: Mining of Electrocardiogram. In: XXI Autumn Meeting of Polish Information Processing Society, Conference Procedings, pp. 169-175 (2005)

11. Acquaticci, F.: Detección de Complejos QRS mediante Redes Neuronales, Buenos Aires Argentina

12. Lagerholm, M., Peterson, C., Braccini, G., Edenbrandt, L., Sörnmo, L.: Clustering ECG Complexes, Using Hermite Functions and Self-Organized Maps. IEEE Transaction on Biomedical Engineering 47(7) (July 2000)

13. Wenyu, Y., Gang, L., Ling, L., Qilian, Y.: ECG Analysis Based on PCA and SOM. In: IEEE Int. Conf. Neural Networks and Signal Processing, Nanjing, China, December 14-17 (2003)

14. Johansson, A.: Neural network for photoplethysmographic respiratory rate monitoring. Medical and Biological Engineering and Computing 41(3), 242-248 (2003)

15. Soltane, M., Ismail, M., Rashid, Z.A.A.: Artificial Neural Networks (ANN) Approach to PPG Signal Classification. International Journal of Computing and Information Sciencies 2(1) (April 2004)

16. Orjuela-Cañón, A., Posada-Quintero, H., Delisle-Rodrıguez, D., Cuadra-Sanz, M., Fernández de la Vara-Prieto, R., López-Delis, A.: Onset and Peak Detection over Pulse Wave Using Supervised SOM Network. International Journal of Bioscience, Biochemistry and Bioinformatics 3(2), 133-137 (2013)

17. Haykin, S.: Neural Networks: A Comprehensive Foundation, 3rd edn. Prentice Hall (1998)

18. Camacho, F.: Statistical Analysis of Central Aortic Blood Pressure Parameters Derived From the Peripheral Pulse, PhD dissertation, University of New South Wales, Sydney (November 2005)

19. Latham, R.D., Westerhof, N., Sipkema, P., Rubal, B.J., Reuderink, P., Murgo, J.P.: Regional wave travel and reflections along the human aorta: A study with six simultaneous micromanometric pressures. Circulation 6, 1257-1269 (1985)

20. Riedmiller, M.: Rprop: Description and Implementation Details. Technical report, University of Karlsruhe (1994)

21. Elisseeff, A.: Leave-one-out error and stability of learning algorithms with applications. Nato Science Series Sub Series III Computer and Systems Sciences 190, 111-130 (2003)

22. Kohavi, R.: A study of cross-validation and bootstrap for accuracy estimation and model selection. In: Proceedings of the 14th International Joint Conference on Artificial Intelligence, pp. 1137-1143 (February 1995)

23. Zoubir, A., Boashash, B.: The Bootstrap and its Application in Signal Processing. IEEE Signal Processing (January 1998)

24. Zoubir, A., Iskander, R.: Bootstrap Techniques for Signal Processing, 1st edn. Cambridge University Press, Cambridge (2004) 